\title{
Malignant hypertension and hyperreninemia: primary or secondary hypertension? A case report
}

\author{
Anna Belfiore, Vincenzo Ostilio Palmieri, Stefania Pugliese, Maria Ciannarella, Antonella Cezza, Piero Portincasa \\ Clinica Medica ‘A. Murri’, Department of Biomedical Sciences \& Human Oncology, University of Bari Medical School, Bari, Italy
}

\begin{abstract}
Malignant hypertension is a rare condition characterized by severe hypertension and multi-organ ischemic damage. Marked activation of the renin-angiotensin system is observed in many patients, but its persistence over time is not known. We report a case of a 42-year-old woman who presented with severe hypertension and multi-organ damage. Initial evaluation showed an elevated value of direct renin concentration with normal plasma aldosterone concentration and a nodular lesion in the left adrenal gland. The differential diagnosis between the primary and secondary form of hypertension had to be questioned. Consequently, the patient was followed up for 20 months. Repeated checks showed a significant increase in renin levels with a normal aldosterone concentration and regression of organ damage. After 20 months, renin values returned within normal range. Hyperreninemia persisting over a long period of time has not been fully explained. Long-term follow-up allowed us to attribute malignant hypertension to de novo essential hypertension.
\end{abstract}

Correspondence: Anna Belfiore, Clinica Medica 'A. Murri', Department of Biomedical Sciences \& Human Oncology, University of Bari Medical School, Bari, Italy.

Fax: +39.080 .5593826 .

E-mail: belfiore.murri@gmail.com

Key words: Malignant hypertension; hyperreninemia; multiorgan damage; primary and secondary hypertension.

Acknowledgments: the authors would like to thank Mrs. Ann Pilkington for her support in reviewing the English text.

Contributions: all authors followed the patient during hospitalization. $\mathrm{AB}$ followed up on the patient during hospitalization and day service; the author made substantial contributions to the interpretation of clinical data and wrote the scientific work. SP followed up the patient during day service and collected the patient's data. VO and PP reviewed the work. MC and $\mathrm{AC}$ worked out the tables and the figure. $\mathrm{MC}$ revised the use of English.

Conflict of interests: the authors declare no potential conflict of interests.

Patient consent: the authors certify that they have obtained the patient consent form. In the form, the patient has given her consent for clinical information to be reported in the journal. The patient understands that her name and initials will not be published, and due efforts will be made to conceal her identity.

Received for publication: 5 November 2020.

Revision received: 29 December 2020.

Accepted for publication: 30 December 2020

This work is licensed under a Creative Commons Attribution NonCommercial 4.0 License (CC BY-NC 4.0).

${ }^{\circ}$ Copyright: the Author(s), 2021

Licensee PAGEPress, Italy

Italian Journal of Medicine 2021; 15:115-119

doi:10.4081/itjm.2021.1419

\section{Introduction}

Malignant hypertension is a rare and severe form of hypertension characterized by a rapid and significant increase in blood pressure and multi-organ ischemic damage with a poor prognosis. The most recent definition emphasizes multi-organ damage with particular reference to retinopathy, acute renal failure, encephalopathy, and thrombotic microangiopathy. ${ }^{1,2}$ The renin-angiotensin system is believed to play a crucial role in the pathogenesis of organ damage. However, the extent of renin-angiotensin activation and its persistence over time is not known.

We report a case of malignant hypertension associated with multi-organ damage and the onset of hypertensive encephalopathy, in which a pronounced elevation in plasma renin levels was observed over a long-term follow-up.

\section{Case Report}

A 42-year-old woman with no prior history of hypertension was admitted to the emergency department of our hospital with headache, nausea, neck pain, and disorders of the visus. She was affected by multiple sclerosis treated with interferon $\beta$ 1a. On admission, her blood pressure was $220 / 150 \mathrm{mmHg}$, pulse 110 beats per minute, and the temperature was $36.5^{\circ} \mathrm{C}$. Suddenly the patient had a generalized tonic-clonic seizure with loss of consciousness. The blood pressure increased to $260 / 150 \mathrm{mmHg}$. The patient was immediately transferred to the Intensive Care Unit and submitted to orotracheal intubation and mechanical ventilation. The 
hypertensive emergency was managed with nitroglycerin infusion and urapidil bolus ev. Brain computed tomography (CT) and then magnetic resonance imaging (MRI) were performed on the same day. CT showed a widespread area of edema affecting the cerebellar hemispheres, the trunk, and the posterior parieto-occipital regions bilaterally. MRI revealed extensive hyperintensity in the same regions and mainly affecting the occipital and parietal lobes on T2-weighted. These findings were suggestive of posterior reversible encephalopathy syndrome (PRES). ${ }^{3}$ In the first days of hospitalization, the patient's blood tests showed a rapid change, as shown in Table 1. On admission to the emergency room, the biochemical parameters were within normal limits. On the second and third day, an increased level of creatinine, mild hypokalemia, anemia, and thrombocytopenia were observed. Anemia associated with the presence of schistocytes on peripheral smear and an increased lactate dehydrogenase suggested ongoing hemolysis. Fibrin degradation products and C-reactive protein (CRP) were also elevated. Renal failure was associated with a proteinuria of $1404 \mathrm{mg}$ over $24 \mathrm{~h}$ and related hypoalbuminemia. After 3 days, the patient was transferred to our internal medicine department. On admission, she was alert, conscious, apyretic with a $150 / 110 \mathrm{mmHg}$ pressure, heart rate of 105 beats per minute, and respiratory rate of 18 acts per minute. Echocardiography showed left ventricular hypertrophy and reduced left ventricular ejection fraction (EF 48\%). On fundus examination, there were retinal hemorrhages and exudates (grade IV hypertensive retinopathy).

These findings were to be referred to as malignant hypertension associated with multi-organ damage. Adequate blood pressure control was achieved using different antihypertensive drugs, such as calcium channel blockers, diuretic, $\beta$-blockers, and angiotensin II receptor antagonists. Interferon- $\beta$ was stopped. Given the severity of clinical manifestations, several diagnostic tests were performed to rule out secondary causes of hypertension. Laboratory tests showed a normal plasma aldosterone concentration (PAC) and elevated value of direct renin concentration (DRC) as shown in Table 2. 24-h urinary excretion of catecholamines and total metanephrines were within the normal range.

Hyperreninemia could be due to the presence of renovascular disease, a renin-secreting tumor, chronic parenchymal renal disease, or a scleroderma renal crisis. The absence of circulating autoantibodies excluded the latter hypothesis. Ultrasonography revealed normal symmetrical kidneys. There was no evidence of renal artery stenosis on Doppler ultrasound. The abdominal and pelvic CT scan showed a hypodense

Table 1. Laboratory data of our patient during hospitalization.

\begin{tabular}{lccc}
\hline & Day $\mathbf{1}$ & Day 3 & Day 12 \\
\hline $\mathrm{Hb}(\mathrm{g} / \mathrm{dL})$ & 13.6 & 8.3 & 10.4 \\
\hline Schistocytes & No & Yes & No \\
\hline Platelets $\left(\mathrm{x} 10^{3} / \mathrm{uL}\right)$ & 76 & 69 & 320 \\
\hline Creatinine $(\mathrm{mg} / \mathrm{dL})$ & 0.73 & 1.32 & 0.75 \\
\hline eGFR $(\mathrm{mL} / \mathrm{min})$ & 102 & 50 & 99 \\
\hline Na+ & 135 & 142 & 145 \\
\hline K+ & 3.4 & 3.1 & 4.2 \\
\hline Total bilirubin $(\mathrm{mg} / \mathrm{dL})$ & 0.2 & 1.32 & 0.29 \\
\hline ASAT $(\mathrm{U} / \mathrm{L})$ & 61 & 49 & 35 \\
\hline ALAT $(\mathrm{U} / \mathrm{L})$ & 124 & 88 & 78 \\
\hline Albuminemia g/dL & 2.3 & 2 & 2.6 \\
\hline LDH $(\mathrm{U} / \mathrm{L})$ & 358 & 466 & 285 \\
\hline Troponin I (ng/mL) & 0.202 & 0.182 & 0.048 \\
\hline NT-proBNP $(\mathrm{pg} / \mathrm{mL})$ & 1258 & 13.617 & 514 \\
\hline D-Dimer & 2023 & 2756 & 534 \\
\hline Fibrinogen $(\mathrm{mg} / \mathrm{dL})$ & 251 & 547 & 234 \\
\hline CRP & 2.9 & 186 & 4 \\
\hline Proteinuria mg/24 h & 1404 & 1827 & 1035 \\
\hline Albuminuria mg/24 h & 764 & 1078 & 600 \\
\hline
\end{tabular}

$\mathrm{Hb}$, haemoglobin; $\mathrm{Na}^{+}$, sodium; $\mathrm{K}+$, potassium; ASAT, aspartate aminotransferase; ALAT, alanine aminotransferase; $\mathrm{LDH}$, lactate dehydrogenase; $\mathrm{CRP}$, C-reactive protein.

Table 2. Values of direct renin concentration, plasma aldosterone concentration, and sodium and potassium status during hospitalization and post-critical phase.

\begin{tabular}{|c|c|c|c|c|c|c|}
\hline & Hospitalization & $4^{\text {th }}$ month & $8^{\text {th }}$ month & $12^{\text {th }}$ month & $16^{\text {th }}$ month & $20^{\text {th }}$ month \\
\hline $\mathrm{DRC}(\mathrm{mU} / \mathrm{L})$ & 407 & 56.1 & 3834 & 2138 & 1135 & 41.6 \\
\hline PAC (ng/dL) & 9.1 & 12.4 & 12.2 & 10.2 & 8.3 & 6.6 \\
\hline $\mathrm{Na}(\mathrm{mEq} / \mathrm{L})$ & 138 & 139 & 137 & 139 & 140 & 138 \\
\hline $\mathrm{K}(\mathrm{mEq} / \mathrm{L})$ & 3.1 & 4.3 & 4.6 & 4.4 & 4.0 & 4.1 \\
\hline 24 h UNaV (mEq/24 h) & 224 & 160 & 128 & 180 & 250 & 179 \\
\hline 24 h UKV (mEq/24 h) & 49 & 40 & 45 & 48 & 72 & 46 \\
\hline
\end{tabular}

DRC, direct renin concentration; PAC, plasma aldosterone concentration; Na, sodium; K, potassium. 
nodular lesion in the left adrenal gland, suggestive of an adenoma. MRI confirmed the finding. Adrenal adenoma was considered an incidentaloma not being compatible with hyperreninemia. Alternatively, the adrenal adenoma could be related to a rare form of primary hyperaldosteronism in which the plasma renin activity is initially elevated and subsequently suppressed. ${ }^{4,5}$ On the other hand, an extrarenal renin-producing tumor is extremely rare. ${ }^{6}$

During hospitalization, progressive reduction in blood pressure values was obtained up to normal level. The second brain MRI showed complete resolution of vasogenic edema in the brain and medulla. Laboratory tests were within normal limits at the time of hospital discharge, although proteinuria persisted. The patient was discharged on the twelfth day in good clinical condition and with an indication to continue the follow-up at our arterial hypertension clinic. Antihypertensive therapy prescribed at discharge included carvedilol $12.5 \mathrm{mg}$ twice a day, amlodipine $10 \mathrm{mg} /$ day, and olmesartan hydrochlorothiazide $20 / 12.5 \mathrm{mg} /$ day.

The follow-up of the patient lasted 20 months. Every 4 months, blood, urine, and instrumental tests were performed. Laboratory tests showed a significant increase in DRC, up to $3834.0 \mathrm{mU} / \mathrm{L}$, with normal blood pressure values. After 20 months from the acute event, renin levels returned within normal limits. Table 2 shows the values of DRC and PAC in the follow-up period; the same table records the values relating to each hormonal sample's sodium and potassium status. Many elements can affect the renin, such as posture, time of day the sample is taken, age, sodium intake, and various antihypertensive drugs. Before each test, olmesartan hydrochlorothiazide was suspended for two weeks and replaced by doxazosin to maintain control of hypertension. The blood sample was obtained in the morning after 60 min sitting rest as recommended by the guidelines. ${ }^{7}$ During the follow-up period, antihypertensive drug therapy was gradually reduced according to reduction and normalization of blood pressure values, as shown in Figure 1. Compliance with antihypertensive therapy was complete as the patient believed in the need for medications and a close follow-up.

Eight months after the acute episode, the fundus exam showed stage 2 hypertensive retinopathy while the echocardiogram highlighted left ventricular hypertrophy regression with normalized ejection fraction, and the 24-hour urine collection showed no proteinuria.

\section{Discussion and Conclusions}

Malignant hypertension is the most severe form of hypertension characterized by a rapid and significant increase over baseline blood pressure associated with multiple organ damage, ${ }^{1,2}$ including bilateral retinal hemorrhages and/or exudates and papilledema, encephalopathy, acute heart failure, and acute deterioration in renal function. Sometimes it is associated with microangiopathic hemolytic anemia. ${ }^{8}$ Clinical manifestations and presentation can be variables in relation to the system mainly involved for each patient. In most cases, malignant hypertension is due to essential hypertension, mainly when untreated or inadequately treated. However, any form of secondary hypertension may progress to malignant hypertension. Secondary causes have been reported in younger patients and include renovascular diseases, renal parenchymal disease, endocrine dysfunction such as pheochromocytoma, primary hyperaldosteronism, renin-secreting tumor. ${ }^{2}$ Differential diagnosis between essential and secondary hypertension is often a challenge, especially during the acute phase of the pathology.

The reported case had all clinical features of malignant hypertension. The onset symptoms were the expression of brain damage as demonstrated by bilateral, T2-hyperintense MRI lesions due to vasogenic edema. The lesions resolved entirely with blood pressure reduction. ${ }^{3}$ In the acute phase of the pathology, the patient presented severe fundus lesions, renal failure including proteinuria, impaired systolic function with left ventricular hypertrophy, and intravascular hemolysis with fragmented red blood cells and consumptions of platelets. The characteristics of anemia associated with coagulation alterations were compatible with microangiopathic hemolytic anemia. ${ }^{8}$ In this context, the increase in CRP observed in our patient in the malignant phase might be explained by the simultaneous activation of coagulation and inflammation. ${ }^{9}$

Malignant hypertension is considered a renin-dependent form of hypertension, but the extent and persistence of renin-angiotensin system activation are remarkably variable and not fully known. In addition, cases without elevated reninemia have been reported. ${ }^{8}$ The activation of the renin-angiotensin system is believed to be due to renovascular ischemia due to severe hypertension and renal arterioles damage ${ }^{8,10}$ Activation of the renin-angiotensin system can trigger a vicious circle, as described by Laragh, causing further vascular damage and a further increase in blood pressure. ${ }^{11}$

The finding of hyperreninemia and its persistence over time were the characterizing elements of the clinical case. High renin malignant hypertension could be related to renovascular disease, chronic parenchymal renal disease, scleroderma renal crisis, renin-secreting tumor, blunt trauma to the kidney; ${ }^{2,12,13}$ alternately, activation of the renin-angiotensin axis could represent the expression of vascular damage induced by a significant elevation of the blood pressure in the transition from benign to malignant hypertension. ${ }^{8,10}$

Given the seriousness of the case, the differential diagnosis between essential form and curable causes of 
hypertension was crucial. For this purpose, the patient underwent a renal duplex ultrasound exam with no evidence of renal artery stenosis. Abdomen and pelvis $\mathrm{CT}$ and MRI showed the presence of a nodular lesion in the left adrenal gland. In the beginning, the lesion was interpreted as an incidentaloma, as the values of aldosterone and urinary catecholamines were normal. However, to confirm this hypothesis and exclude rare forms of hyperaldosteronism with increased renin levels in the malignant phase as previously described by other authors, ${ }^{4,5}$ were repeated the hormonal dosages in the following months. Repeated laboratory tests at intervals of 4 months showed a significant increase in DRC dissociated from plasma aldosterone values (Table 2) and pressure values (Figure 1). The value of DRC was normal in the $20^{\text {th }}$ month.

\section{$B P$ and DRC trend}

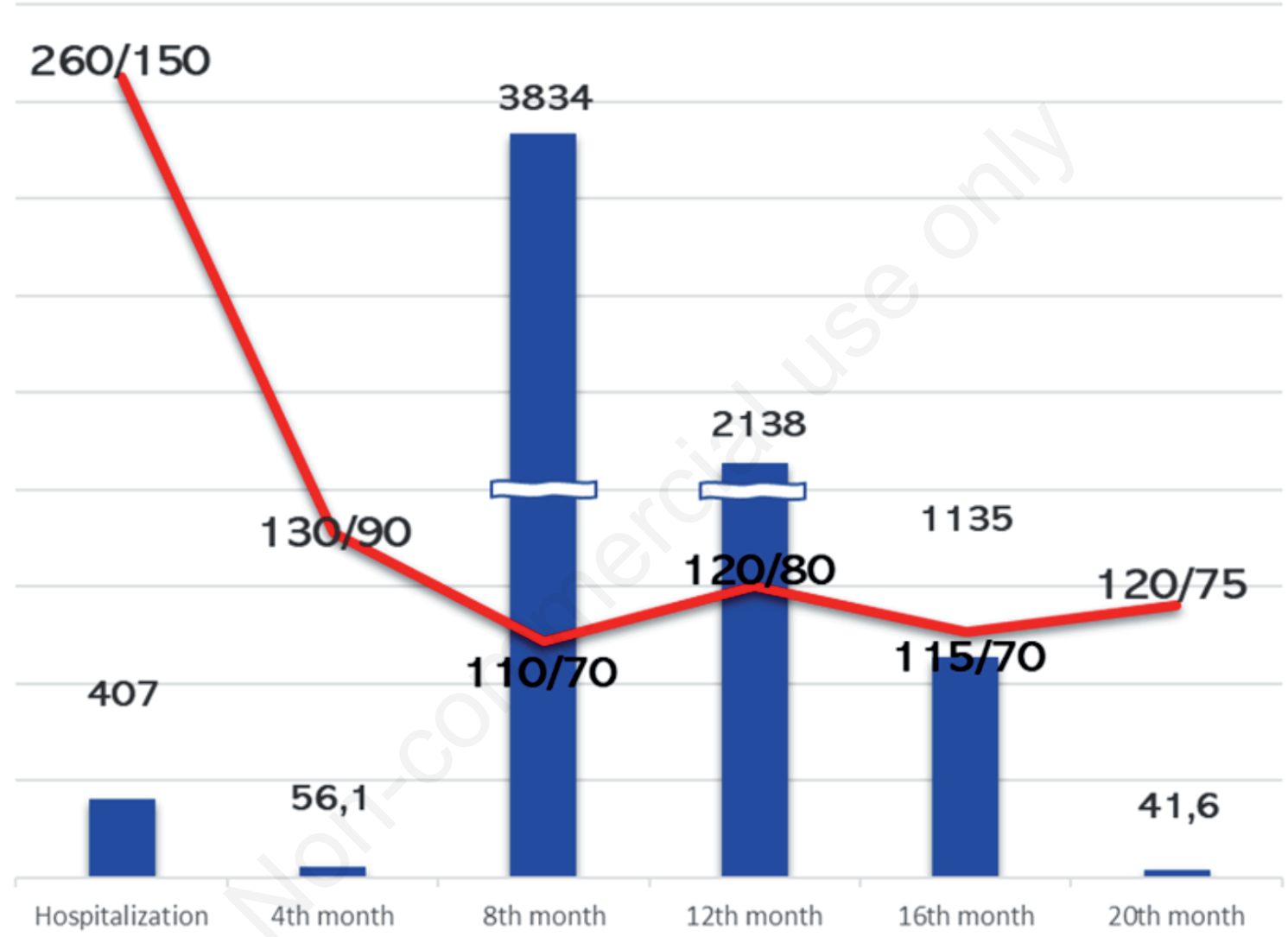

\section{Therapy}

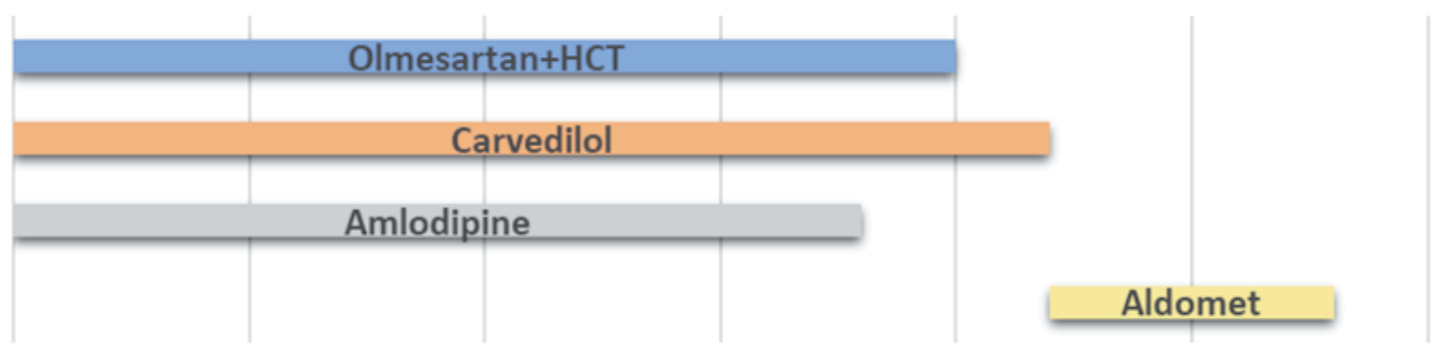

Figure 1. Clinical course: mean arterial pressure (red line), direct renin concentration (blue columns), and antihypertensive therapy over the 20-months follow-up. BP, blood pressure; DRC, direct renin concentration; HCT, hydrochlorothiazide. 
Several antihypertensive drugs can give rise to hyperreninemia, including diuretics, ACE-inhibitors, angiotensin II receptor blockers (ARBs), dihydropyridine calcium channel blockers, and aldosterone antagonists. ${ }^{14}$ The patient was on ARBs therapy, which effectively lowered blood pressure associated with the other drugs. It is not known whether the ARBs long-term effects may have contributed to the persistence of such elevated renin levels, as observed in our patient.

In the months preceding the last dosage of DRC, the ongoing antihypertensive therapy was stopped, and alpha methyldopa was started at the request of the patient who wanted to face pregnancy and avoid potentially harmful drugs. Alfa methyldopa is known to decrease renin level, ${ }^{15,16}$ but we do not believe this could explain the sudden changes in renin level observed in our patient. The DRC was not measured in balance on sodium diet, but the value of sodium concentration in the blood and urine was always within the norm's limits (Table 2). As shown by the values included in the table, the renin levels are independent of sodium and potassium status.

The lack of DRC suppression over a long period of time in the post-malignant phase has not been fully explained. In addition, in our patient, we observed organ damage recovery in the first months of follow-up. Several authors have shown a correlation between renal microvascular damage and renin hypersecretion in patients with malignant hypertension..$^{8,17,18}$ Our patient did not undergo kidney biopsy due to the rapid improvement in renal function; therefore, it was impossible to correlate renin levels with renal microvascular injury.

Based on clinical and laboratory findings and after long-term follow-up, we concluded that our patient had de novo primary hypertension complicated by a malignant phase. The long follow-up was to ensure that the DRC and PAC discrepancy in the post-malignant phase was not due to secondary forms of hypertension. In any case, clinical and laboratory monitoring of the patient persisted.

In conclusion, this case highlights the role of reninangiotensin system activation as a key factor to malignant hypertension pathogenesis and the difficulty in differentiating between primary and secondary hypertension in the malignant phase. Furthermore, this case stresses the role of adequate follow-up of all patients in the post-critical phase, to exclude secondary form of hypertension, evaluate the regression of organ damage, and monitor renin and aldosterone levels.

\section{References}

1. Cremer A, Amraoui F, Lip GYH, et al. From malignant hypertension to hypertension-MOD: a modern definition for an old but still dangerous emergency. J Hum Hypertens 2016;30:463-6.
2. van den Born B-JH, Lip GYH, Brguljan-Hitij J, et al. ESC Council on hypertension position document on the management of hypertensive emergencies. Eur Heart J Cardiovasc Pharmacother 2019;5:37-46.

3. Fugate JE, Rabinstein AA. Posterior reversible encephalopathy syndrome: clinical and radiological manifestations, pathophysiology, and outstanding questions. Lancet Neurol 2015;14:914-25.

4. Oka K, Hayashi K, Nakazato T, et al. Malignant hypertension in a patient with primary aldosteronism with elevated active renin concentration. Intern Med 1997;36:700-4.

5. Maruhashi T, Amioka M, Kishimoto S, et al. Elevated plasma renin activity caused by accelerated-malignant hypertension in a patient with aldosterone-producing adenoma complicated with Renal Insufficiency. Intern Med 2019; 58:3107-11.

6. Iimura O, Shimamoto K, Hotta D, et al. A Case of adrenal tumor producing renin, aldosterone, and sex steroid hormones. Hypertension 1986;8:951-6.

7. Rossi GP, Bisogni V, Bacca AV, et al. The 2020 Italian Society of Arterial Hypertension (SIIA) practical guidelines for the management of primary aldosteronism. Int J Cardiol Hypertens 2020;5:1000292.

8. van den Born B-JH, Koopmans RP, Van Montfrans GA. The renin-angiotensin system in malignant hypertension revisited: plasma renin activity, microangiopathic hemolysis, and renal failure in malignant hypertension. Am J Hypertens 2007;20:900-6.

9. Derhaschnig U, Testori C, Riedmueller E, et al. Hypertensive emergencies are associated with elevated markers of inflammation, coagulation, platelet activation and fibrinolysis. J Hum Hypertens 2013; 27:368-73.

10. Vaughan CJ, Delanty N. Hypertensive emergencies. Lancet 2000;356:411-7.

11. Laragh JH, Baer L, Brunner HR, et al. Renin, angiotensin and aldosterone system in pathogenesis and management of hypertensive vascular disease. Am J Med 52:633-52.

12. van den Born B-JH, Koopmans RP, Groeneveld JO, van Montfrans GA. Ethnic disparities in the incidence, presentation and complications of malignant hypertension. J Hypertens 2006;24:2299-304.

13. McCune TR, Stone WJ, Breyer JA. Page kidney: case report and review of the literature. Am J Kidney Dis 1991;18:593-9.

14. Mulatero P, Rabbia F, Milan A, et al. Drug effects on aldosterone/plasma renin activity ratio in primary aldosteronism. Hypertension 2002;40:897-902.

15. Mohammed S, Fasola AF, Privitera PJ, et al. Effect of methyldopa on plasma renin activity in man. Circ Res 1969;25:543-8.

16. Weidman P, Hirsch D, Maxwell MH, et al. Plasma renin and blood pressure during treatment with methyldopa. Am J Cardiol 1974;34:671-6.

17. McAllister RG, Michelakis AM, Oates JA, Foster JH. Malignant hypertension due to renal artery stenosis. Greater renin release from the nonstenotic kidney. JAMA 1972;221:865-8.

18. McLaren KM, MacDonald MK. Histological and ultrastructural studies of the human juxtaglomerular apparatus in benign and malignant hypertension. J Pathol 1983;139:41-55. 